This item was submitted to Loughborough's Research Repository by the author.

Items in Figshare are protected by copyright, with all rights reserved, unless otherwise indicated.

\title{
Asymptotic decay of pair correlations in a Yukawa fluid
}

PLEASE CITE THE PUBLISHED VERSION

http://dx.doi.org/10.1103/PhysRevE.71.027401

\section{PUBLISHER}

(c) The American Physical Society

\section{VERSION}

VoR (Version of Record)

\section{PUBLISHER STATEMENT}

This work is made available according to the conditions of the Creative Commons Attribution-NonCommercialNoDerivatives 4.0 International (CC BY-NC-ND 4.0) licence. Full details of this licence are available at: https://creativecommons.org/licenses/by-nc-nd/4.0/

\section{LICENCE}

CC BY-NC-ND 4.0

\section{REPOSITORY RECORD}

Hopkins, Paul, Andrew J. Archer, and Robert Evans. 2019. "Asymptotic Decay of Pair Correlations in a Yukawa Fluid”. figshare. https://hdl.handle.net/2134/16039. 


\title{
Asymptotic decay of pair correlations in a Yukawa fluid
}

\author{
P. Hopkins, A. J. Archer, ${ }^{*}$ and R. Evans \\ H. H. Wills Physics Laboratory, University of Bristol, Bristol BS8 1TL, United Kingdom
}

(Received 27 August 2004; published 23 February 2005)

\begin{abstract}
We analyze the $r \rightarrow \infty$ asymptotic decay of the total correlation function $h(r)$ for a fluid composed of particles interacting via a (point) Yukawa pair potential. Such a potential provides a simple model for dusty plasmas. The asymptotic decay is determined by the poles of the liquid structure factor in the complex plane. We use the hypernetted-chain closure to the Ornstein-Zernike equation to determine the line in the phase diagram, well removed from the freezing transition line, where crossover occurs in the ultimate decay of $h(r)$, from monotonic to damped oscillatory. We show that (i) crossover takes place via the same mechanism (coalescence of imaginary poles) as in the classical one-component plasma and in other models of Coulomb fluids and (ii) leading-order pole contributions provide an accurate description of $h(r)$ at intermediate distances $r$ as well as at long range.
\end{abstract}

DOI: 10.1103/PhysRevE.71.027401

PACS number(s): 52.27.Lw, 61.20.Ne, 05.20.Jj

The Yukawa, or screened Coulomb, potential is often used as a model for fluids composed of charged particles immersed in a uniform neutralizing background. We define the pair potential as

$$
\phi(r)=\frac{\epsilon \exp (-\lambda r)}{\lambda r},
$$

where $r$ is the distance between the centers of a pair of particles, $\lambda$ is the inverse decay length (screening parameter), and $\epsilon>0$ characterizes the strength of the potential. A fluid composed of particles interacting via this potential exhibits behavior similar to that of the one-component classical plasma (OCP) $[1,2]$, in which $\phi(r) \propto 1 / r$. The point Yukawa pair potential also corresponds to a limiting case of the well established Derjaguin, Landau, Verwey and Overbeek (DLVO) model for aqueous suspensions of charged colloidal particles [3-5], where the hard-core part of the colloidcolloid effective pair potential is neglected.

The Yukawa pair potential is also widely employed in theoretical studies of the so-called dusty plasmas. These are multicomponent plasmas consisting of charged (dust) particles, electrons, and ions, as well as neutral atoms or molecules, which are found in a variety of environments, from the interstellar medium to plasma etching processes. Depending on their size, the dust grains can attain a large negative charge of $1000 e-10000 e$ for particles of size 1 $-10 \mu \mathrm{m}$ [6]; the charge is generally negative and is determined by the balance of the absorbed electron and ion fluxes. Since the dust component of the plasma can be videoed and tracked directly, dusty plasmas provide a valuable system for studying both equilibrium phenomena and collective processes such as transport in a fluid [6,7]. In modeling the dusty plasma, the effective potential between two dust particles of charge $Q$ is taken to be $[8,9]$

$$
\phi(r)=\frac{Q^{2}}{4 \pi \epsilon_{0} r} \exp \left(-k_{D} r\right),
$$

where $k_{D}^{-1}$ is the Debye length of the background plasma. The thermodynamics of the system can then be characterized by the dimensionless parameters

\footnotetext{
*Electronic address: Andrew.Archer@bristol.ac.uk
}

$$
\kappa=k_{D} a \quad \text { and } \quad \Gamma=\frac{Q^{2}}{4 \pi \epsilon_{0} a k_{B} T},
$$

where $a \equiv[3 /(4 \pi \rho)]^{1 / 3}$ is the Wigner-Seitz radius, i.e., the mean interparticle distance, which is determined by the average fluid number density $\rho . \Gamma$ is the coupling (plasma) parameter [9]. Note that unlike the OCP, whose properties depend solely on $\Gamma$, two parameters are required in this case.

Because of its relevance for colloidal fluids and the growing importance of studies of dusty plasmas, not to mention the appealing mathematical properties of the Yukawa potential [10], much is established for this model fluid. The phase diagram has been determined in a number of simulation studies [11-16]. At small values of $\Gamma$ the liquid state equilibrium structure is well approximated by the hypernetted-chain (HNC) closure to the Ornstein-Zernike (OZ) equation [17-19], as one would expect from studies of the OCP [1]. For larger values of $\Gamma$, a modified HNC based upon a rescaling of the bridge function for the OCP [20] yields results indistinguishable from Monte Carlo (MC) simulation data [17]. At small values of $\kappa$ the Yukawa fluid freezes into a bcc crystal upon increasing $\Gamma$, as in the OCP. For sufficiently large $\kappa$ increasing $\Gamma$ can lead to freezing directly into a fcc crystal. A portion of the phase diagram from Ref. [16] is shown in Fig. 1 [21].

In the present work we analyze the asymptotic decay of the pair correlations in the uniform fluid and we find that at couplings $\Gamma$ below freezing, there is a crossover in the form of the asymptotic decay, $r \rightarrow \infty$, of $h(r)$, the total correlation function, from monotonic to damped oscillatory, that is similar to the crossover found near $\Gamma_{K}=1.12$ in the HNC treatment of the OCP [22]. We map out the crossover line in the $(\kappa, \Gamma)$ phase diagram; see Fig. 1 .

Our starting point is the OZ equation [19], which relates $h(r)$ to $c(r)$, the pair direct correlation function. In Fourier space the $\mathrm{OZ}$ equation can be written as

$$
\hat{h}(q)=\frac{\hat{c}(q)}{1-\rho \hat{c}(q)} .
$$

Here $\hat{f}(q)$ denotes the three-dimensional Fourier transform of the spherically symmetric function $f(r)$. We choose to imple- 


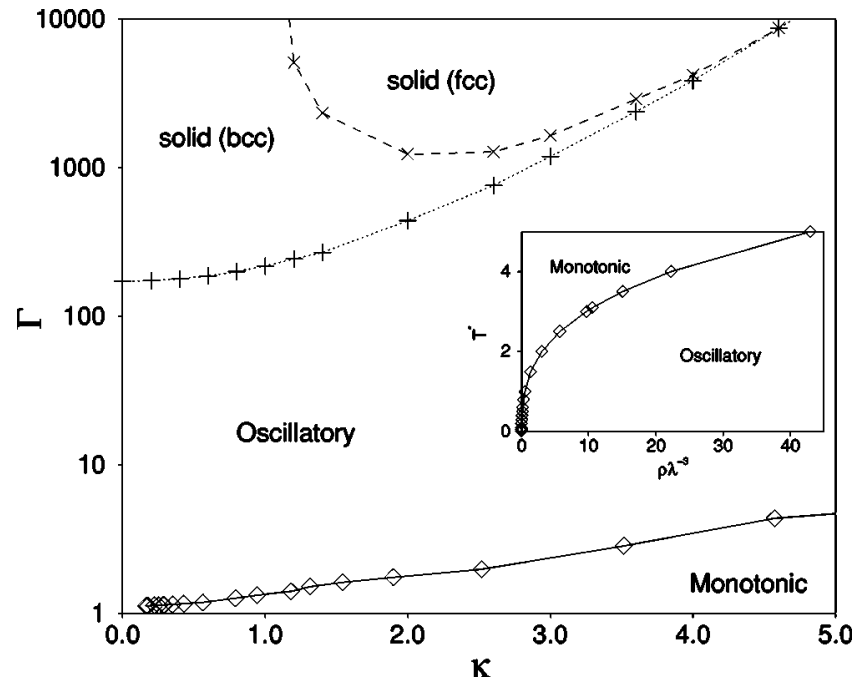

FIG. 1. Crossover line (diamonds joined by a solid line) separating the region of the $(\kappa, \Gamma)$ plane where the asymptotic decay of $h(r)$ is damped oscillatory from that where it is monotonic. In the OCP, corresponding to $\kappa=0$, crossover occurs at $\Gamma_{K}=1.21$ [22]. We also display the fluid-solid $(+)$ and solid-solid $(X)$ phase boundaries given in Ref. [16]. In the inset we display the crossover line in the $(\rho, T)$ plane. Note that the freezing transition present at low $T^{*} \equiv k_{B} T / \epsilon$ is not visible on this scale.

ment the HNC closure which sets the bridge function $B(r)$ $=0$. For the present Yukawa fluid, particularly at the densities in the neighborhood of the crossover line, the HNC accounts very well for the structure of the uniform fluid, yielding pair correlation functions almost indistinguishable from simulation results. Thus we shall use the HNC results to determine the location of the crossover line. First, in Fig. 2(a) we display some typical results for $g(r)=1+h(r)$. These are for a fixed temperature $T^{*} \equiv k_{B} T / \epsilon=1$ and for the densities $\rho \lambda^{-3}$ $=0.1,0.45,2.5,10$, and 40. At this temperature, we shall find that the crossover occurs for $\rho \lambda^{-3} \simeq 0.47$. We also display some constant NVT MC results for the first three of these densities; for $\rho \lambda^{-3}=0.1$ and 0.45 we used 300 particles with 25000 (plus 10000 equilibration) trial moves per particle. The maximum displacement of the particles was chosen so that approximately $50 \%$ of attempted moves were accepted. For the MC simulations at $\rho \lambda^{-3}=2.5$ we used 2000 particles. We see that as the density is increased the asymptotic decay switches from monotonic to damped oscillatory. This crossover represents the evolution of the system from a weakly coupled state where the particles do not order strongly, to a state where they are more closely packed and the correlations become more hard-sphere-like, although for all the densities displayed, the amplitude of the (oscillatory) structure in $g(r)$ remains quite small.

The asymptotic decay of $h(r)$ can be obtained [22,23] from the $\mathrm{OZ}$ equation using the inverse Fourier transform of Eq. (4):

$$
r h(r)=\frac{1}{4 \pi^{2} i} \int_{-\infty}^{\infty} d q q \exp (\text { iqr }) \frac{\hat{c}(q)}{1-\rho \hat{c}(q)},
$$

which can be transformed into a semicircular contour integral in the upper half of the complex plane. Its value is determined by the poles of $\hat{h}(q)$ enclosed. These occur at $q_{n}= \pm \alpha_{1}+i \alpha_{0}$, where $q_{n}$ is the solution to the equation

$$
1-\rho \hat{c}\left(q_{n}\right)=0 \text {. }
$$

As a result, $h(r)$ can be obtained as the sum $[22,23]$

$$
r h(r)=\frac{1}{2 \pi} \sum_{n} R_{n} \exp \left(i q_{n} r\right),
$$

where $q_{n}$ is the $n$th pole and $R_{n}$ is the residue of $q \hat{c}(q)$ $/[1-\rho \hat{c}(q)]$ at $q_{n}$. Clearly the asymptotic behavior of $h(r)$ is determined by the pole(s) with the smallest imaginary part, $\alpha_{0}$. If this pole is purely imaginary, $q_{n}=i \alpha_{0}$, then $r h(r)$ $\sim A \exp \left(-\alpha_{0} r\right)$, for $r \rightarrow \infty$, where $A$ is a (real) amplitude [23]. Alternatively, if the conjugate pair $q_{n}= \pm \alpha_{1}+i \widetilde{\alpha}_{0}$ has the smallest imaginary part then $r h(r) \sim \widetilde{A} \exp \left(-\widetilde{\alpha}_{0} r\right)$ $\times \cos \left(\alpha_{1} r-\theta\right)$. The amplitudes $A$ and $\tilde{A}$ and the phase $\theta$ can be calculated from the residues [23]. Whether a pure imaginary or complex pole dominates depends on the thermodynamic state point.

In order to calculate the poles, we use the separation method introduced in Ref. [22]. Owing to the particular form of the decay of the Yukawa pair potential, the asymptotic behavior of $c(r)$ must be treated separately so as to ensure the convergence of the integrals which determine the poles [22]. The asymptotic decay $r \rightarrow \infty$ of the direct correlation function is given by $c(r) \sim-\beta \phi(r)$, which for the Yukawa potential implies $c(r) \sim-\exp (-\lambda r) /\left(T^{*} \lambda r\right)$. It is convenient to define a short-ranged direct correlation function $c^{s r}(r)$ by subtracting the long-ranged Yukawa decay. The Fourier transform of $c(r)$ is then

$$
\hat{c}(q) \equiv \hat{c}^{s r}(q)-\frac{4 \pi}{\lambda T^{*}} \frac{1}{\left(q^{2}+\lambda^{2}\right)} .
$$

Making this division, we follow Ref. [22] and calculate the poles by separating Eq. (6) into its real and imaginary parts and solving numerically using a Newton-Raphson procedure. However, in general the integrals involved converge only for complex $q$ such that $\operatorname{Im}[q]<2 \alpha_{0}$, where $\alpha_{0}$ is the imaginary part of the leading order pole, i.e., that with the smallest value of $\alpha_{0}$. In practice, the other poles generally lie outside this region of convergence and only the leading-order pole can be determined. We also use this separation of $c(r)$ to calculate the amplitude and phase of $h(r)$ from the residues of the poles, assuming these to be simple [22].

Using our HNC results for $c(r)$ we were able to calculate the contributions to $h(r)$ from the leading order pole(s) for various points in the phase diagram. In Fig. 2(b) we display the HNC result for $h(r)$ at the state point $\rho \lambda^{-3}=0.1$ and $T^{*}$ $=1$, together with the contribution from the leading-order (purely imaginary) pole. This state point lies on the monotonic side of the crossover line. In Fig. 2(c) we display the HNC result for $h(r)$ at $\rho \lambda^{-3}=0.45$ and $T^{*}=1$ (near the crossover line, still on the monotonic side), together with the contributions from the two leading-order (purely imaginary) poles. In Fig. 2(d) we display the HNC result for $h(r)$, for the state point $\rho \lambda^{-3}=40$ and $T^{*}=1$, which exhibits damped oscillatory asymptotic decay, together with the contribution 

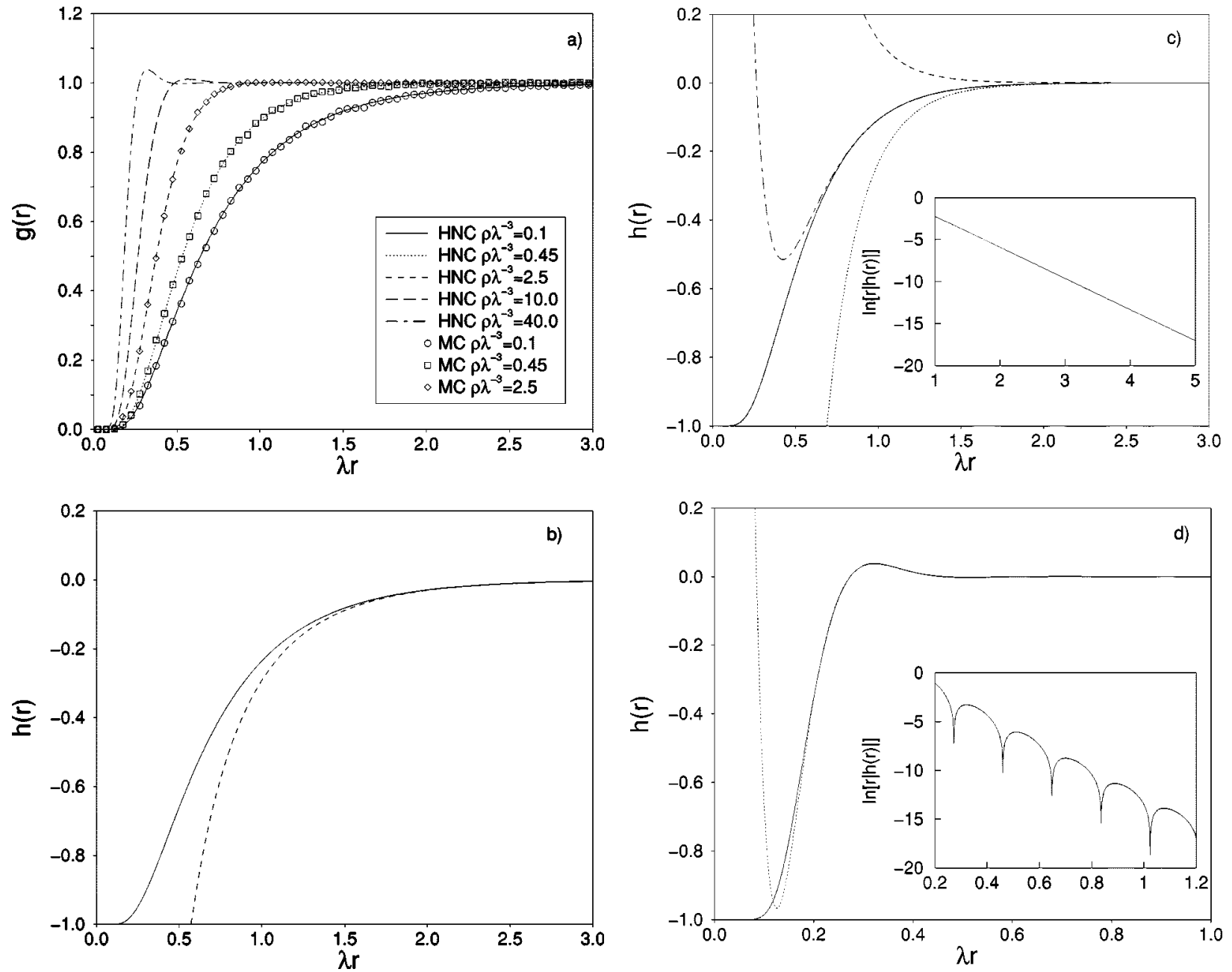

FIG. 2. Pair correlation functions for a reduced temperature $T^{*}=1$. (a) $g(r)$ calculated for a range of densities; the lines denote HNC results and the symbols MC results (see legend). Crossover occurs at $\rho \lambda^{-3} \simeq 0.47$ for this temperature. For densities larger than this the decay of $g(r)$ is damped oscillatory. (b) Comparison between the full $\mathrm{HNC}$ result for $h(r)$ (solid line) and the contribution from the leading-order single imaginary pole (dashed line) for $\rho \lambda^{-3}=0.1$. (c) The full $\mathrm{HNC}$ result for $h(r)$ (solid line) and the contributions from the two leading-order purely imaginary poles (dotted and dashed lines) and their sum (dot-dashed line), for $\rho \lambda^{-3}=0.45$. (d) The full HNC result for $h(r)$ (solid line) and the contribution from the leading-order conjugate pair of complex poles (dotted line), giving damped oscillatory decay, for $\rho \lambda^{-3}=40$. The insets in (c) and (d) show $\ln [r|h(r)|]$ versus $\lambda r$.

from the leading-order conjugate pair of complex poles. Note that the contribution from this pair of poles approximates accurately the full $h(r)$ for $\lambda r>0.2$.

In Fig. 3 we display the leading-order poles calculated along the isotherm $T^{*}=1$. At low densities the pole dominating the decay of $h(r)$ is purely imaginary. However, as the density is increased, this pole moves up the imaginary axis, and at a density $\rho \lambda^{-3} \simeq 0.47$ it meets a second (descending) purely imaginary pole. This second pole could only be determined once it had descended into the region of convergence. These poles coalesce and form a conjugate pair of complex poles which then move away from the imaginary axis as the density is increased further. This coalescence of two purely imaginary poles to form a pair of complex poles as one moves along a path of increasing density in the phase diagram results in a crossover in the asymptotic decay of $h(r)$ from monotonic to damped oscillatory. The mechanism of poles coalescing and moving off the imaginary axis is the same as that found in the OCP near $\Gamma_{K}=1.12$ [22]. It is equivalent to that found in studies of charge correlations in the restricted primitive model (RPM) of binary ionic fluids [24] and in screened versions of the RPM [25]. Kirkwood [26] was the first to describe the mechanism, so the line in the phase diagram at which crossover occurs could be termed the Kirkwood line following earlier terminology [22,24,25].

In Fig. 1 we display the location of the crossover (Kirkwood) line in the phase diagram. This line (see inset) was determined by finding the density at which the imaginary poles coalesce for a series of isotherms, i.e., fixed $T^{*}$. By converting from the variables $\left(\rho \lambda^{-3}, T^{*}\right)$ to $(\kappa, \Gamma)$ the crossover line shown in the main figure was obtained. We emphasize that this line lies far below the fluid-solid transition line, as might have been expected from the observation that in the OCP freezing is known to occur for $\Gamma \simeq 172$, whereas crossover from monotonic to oscillatory decay of correlations occurs near $\Gamma_{K}=1.12$. For the values of $\kappa$ considered here, 0 


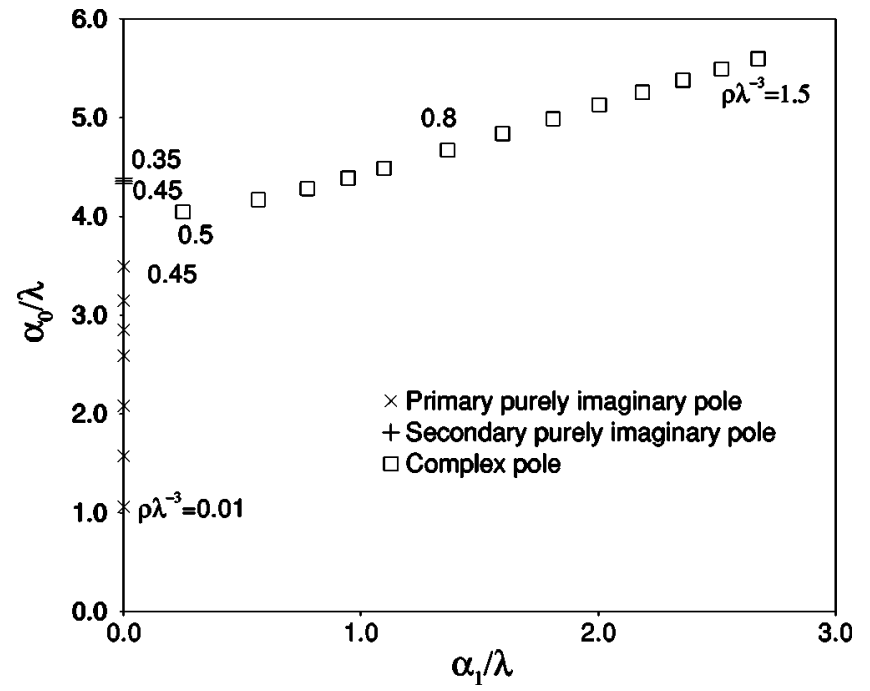

FIG. 3. The leading-order poles (smallest imaginary part, $\alpha_{0}$ ) along the isotherm $T^{*}=1$ for increasing $\rho \lambda^{-3}$. Only poles with positive $\alpha_{1}$ are shown; complex poles occur in conjugate pairs. At low densities the leading order pole $(X)$ is purely imaginary. As the density is increased a second purely imaginary pole $(+)$ descends and, at a density $\rho \lambda^{-3} \simeq 0.47$, the two purely imaginary poles coalesce to form a pair of complex poles $(\square)$. At this (Kirkwood) point the asymptotic decay of $h(r)$ crosses over from monotonic to damped oscillatory. $\leqslant \kappa \lesssim 5$, crossover occurs for $\Gamma$ in the range $1 \lesssim \Gamma \lesssim 5$, i.e., the coupling parameter is rather weak and we expect the HNC to perform rather well; see also Fig. 2(a) for the comparison with $\mathrm{MC}$ results. Of course, one could attempt to improve upon the HNC by incorporating an approximate bridge function $B(r)$, along the lines of Refs. [17,18]. However, we do not expect significant changes in the location of the crossover line; the poles should not be sensitive to details of the particular closure [22]. One could also attempt to extract the poles and determine the crossover line using accurate simulation data for $g(r)$ following the method applied in Ref. [27] for a truncated Lennard-Jones fluid.

In summary, we have shown that the form of the asymptotic decay of the total correlation function $h(r)$ in a Yukawa fluid crosses over from monotonic at small coupling parameter $\Gamma$ to damped oscillatory at larger values via the same mechanism as in the OCP. We find that leading-order asymptotics provide as accurate a description of pair correlations at intermediate range in the Yukawa fluid as they do for other model fluids [22-25,27]. This observation might prove useful in further applications of the Yukawa model to dusty plasmas.

A.J.A. gratefully acknowledges the support of EPSRC under Grant No. GR/S28631/01. R.E. thanks M. Dijkstra and $\mathrm{R}$. Roth for instructive discussions.
[1] M. Baus and J.-P. Hansen Phys. Rep. 59, 1 (1980).

[2] Y. Levin, Rep. Prog. Phys. 65, 1577 (2002).

[3] J-P. Hansen and H. Löwen, Annu. Rev. Phys. Chem. 51, 209 (2000).

[4] A. P. Hynninen and M. Dijkstra, Phys. Rev. E 68, 021407 (2003).

[5] R. Klein et al., J. Phys.: Condens. Matter 14, 7631 (2002).

[6] For a recent review see A. Piel and A. Melzer, Adv. Space Res. 29, 1255 (2002), and references therein.

[7] O. Vaulina et al., Phys. Rev. E 66, 016404 (2002).

[8] Y. Rosenfeld, Phys. Rev. E 49, 4425 (1994).

[9] R. T. Farouki and S. Hamaguchi, J. Chem. Phys. 101, 9885 (1994)

[10] J. S. Rowlinson, Physica A 156, 15 (1989).

[11] K. Kremer et al., Phys. Rev. Lett. 57, 2694 (1986).

[12] M. O. Robbins et al., J. Chem. Phys. 88, 3286 (1988).

[13] R. O. Rosenberg and D. Thirumalai, Phys. Rev. A 36, 5690 (1987).

[14] E. J. Meijer and D. Frenkel, J. Chem. Phys. 94, 2269 (1991).

[15] S. Hamaguchi et al., J. Chem. Phys. 105, 7641 (1996).

[16] S. Hamaguchi et al., Phys. Rev. E 56, 4671 (1997). These authors find the fluid-fcc-bcc triple point at $\kappa=4.28$ and $\Gamma$ $=5.6 \times 10^{3}$ and freezing of the OCP $(\kappa=0)$ at $\Gamma=172$.

[17] See, for example, W. Daughton et al., Phys. Rev. E 61, 2129 (2000), and references therein.

[18] Y. Rosenfeld and N. W. Ashcroft, Phys. Rev. A 20, 1208 (1979).
[19] J-P. Hansen and I. R. McDonald, Theory of Simple Liquids, 2nd ed. (Academic, London, 1986).

[20] H. Iyetomi, S. Ogata, and S. Ichimaru, Phys. Rev. A 46, 1051 (1992).

[21] Note that much of the impetus for studying the phase diagram of the Yukawa fluid stems from laboratory observations of crystalline ordering in dusty plasmas at large coupling $\Gamma$ $[6,7,16]$.

[22] R. J. F. Leote de Carvalho et al., Phys. Rev. E 59, 1435 (1999), and references therein.

[23] R. Evans et al., J. Chem. Phys. 100, 591 (1994).

[24] R. J. F. Leote de Carvalho and R. Evans, Mol. Phys. 83, 619 (1994).

[25] R. J. F. Leote de Carvalho and R. Evans, Mol. Phys. 92, 211 (1997).

[26] See J. G. Kirkwood, Chem. Rev. (Washington, D.C.) 19, 275 (1936), who discussed the potential of mean force in strong electrolytes.

[27] M. Dijkstra and R. Evans, J. Chem. Phys. 112, 1449 (2000). These authors calculated the so-called Fisher-Widom line which separates a region of the phase diagram where $h(r)$ decays monotonically from one where the decay is damped oscillatory. Note that this crossover behavior does not correspond to coalescing of imaginary poles. Rather it is associated with a different mechanism [23] that relies upon the presence of a liquid-gas spinodal, not present for the Yukawa fluid considered here. 